\title{
A quasi-dynamic optimal control strategy for non-linear multi-variable processes based upon non-quadratic objective functionals
}

\author{
JENS G. BALCHEN $\dagger$
}

Keywords: optimal control, steady state optimization, non-quadratic objective functions, LQG-control.

The problem of systematic derivation of a quasi-dynamic optimal control strategy for a non-linear dynamic process based upon a non-quadratic objective function is investigated. The wellknown LQG-control algorithm does not lead to an optimal solution when the process disturbances have non-zero mean. The relationships between the proposed control algorithm and LQG-control are presented. The problem of how to constrain process variables by means of 'penalty'terms in the objective function is dealt with separately.

\section{Introduction}

Optimal control of non-linear multivariable processes with arbitrary objective functionals can be derived using Pontryagin's Maximum Principle (Pontryagin 1962) or Dynamic Programming (Bellman 1965). In most cases of industrial process control, the computational effort associated with applying either of these two methods turns out to be prohibitive. In the case of the Maximum Principle, the reason lies in the fact that a Two Point Boundary Value Problem (TPBVP) has to be solved requiring iterative solutions. In Dynamic Programming the dimensionality problem makes practical applications difficult.

In most cases of industrial process control one would tend to be satisfied with a less stringent solution which yields optimality under somewhat relaxed conditions. If the problem initially is defined properly, one may find a strategy of quasi-dynamic optimal control which under certain assumptions yields a performance which is close to the optimal, but non-practical solution.

The method proposed in this paper is motivated by the desire to find a control strategy which optimizes some realistic profit function for the process (earnings minus cost) in an average sense (steady state) and also gives an adequate supression of the effects of transient process disturbances in a minimum variance sense. The quasi-dynamic optimal control-strategy thus contains two parallel strategies operating simultaneously, one active on low frequency disturbances and the other active on high frequency disturbances. In comparison with conventional process control schemes the low frequency control strategy will be equivalent to integral action except the goal is not to drive the control deviation to zero, but rather to force the process to an optimum.

Received November 1984

† Division of Engineering Cybernetics, The Norwegian Institute of Technology, Trondheim, Norway. 


\section{Problem formulation}

In many continuous industrial processes for which dynamic, multivariable control is desirable, the need for control comes primarily from disturbances acting upon the process. Often such disturbances can be approximated by a sum of two kinds of disturbances, namely a slow drift and a relatively fast (nearly white noise) fluctuation around the drift. Based upon this idea, we now assume that the disturbances acting upon the process can be described by the vector

$$
\boldsymbol{v} \cong \overline{\boldsymbol{v}}+\Delta \boldsymbol{v}
$$

in which $\bar{v}$ represents the slowly varying drift and $\Delta v$ is the fast random fluctuation as indicated for a scalar case in Fig. 1.

The problem is now formulated as follows. The process is described by

where

$$
\dot{x}=f(x, u, v)
$$

$\boldsymbol{x}$ : state vector

$\boldsymbol{u}$ : control vector

$\boldsymbol{v}$ : disturbance vector

$f(\cdot)$ : vector of nonlinear functions

and

where

$$
y=g(x, w)
$$

$\boldsymbol{y}$ : measurement vector

$\boldsymbol{w}$ : measurement noise vector

$g(\cdot)$ : vector of nonlinear measurement functions

The process of (2) is to be controlled via the control vector $(u)$ in such a way that the objective function $L_{1}(x, u)$ is minimized in the steady state, and the mean of the objective function $L_{2}(x, u)$ (an objective functional) is maximized with reference to the dynamic disturbances.

Since according to (3) the state vector is not directly measurable, it is necessary first to consider the problem of state estimation so as to derive an estimated state vector $\hat{\boldsymbol{x}}$ which can be used in the calculations.

Figure 2 is a block diagram of a state estimator based upon (2) and (3). The estimator has the form of an Extended Kalman Filter (EKF). It includes a model of the slowly varying disturbance (drift) given by the equation

$$
\dot{\vec{v}}=\boldsymbol{O}
$$

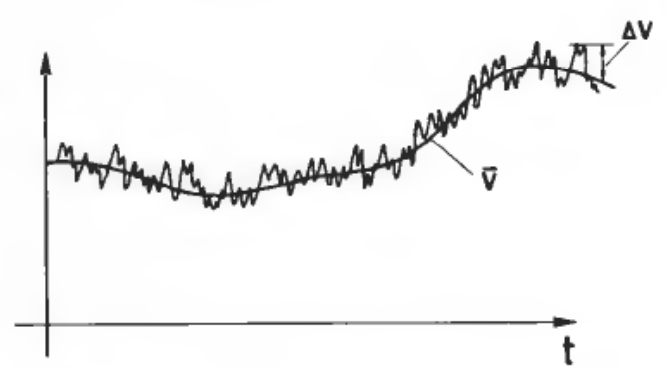

Figure 1. The fast and slow components of a disturbance. 


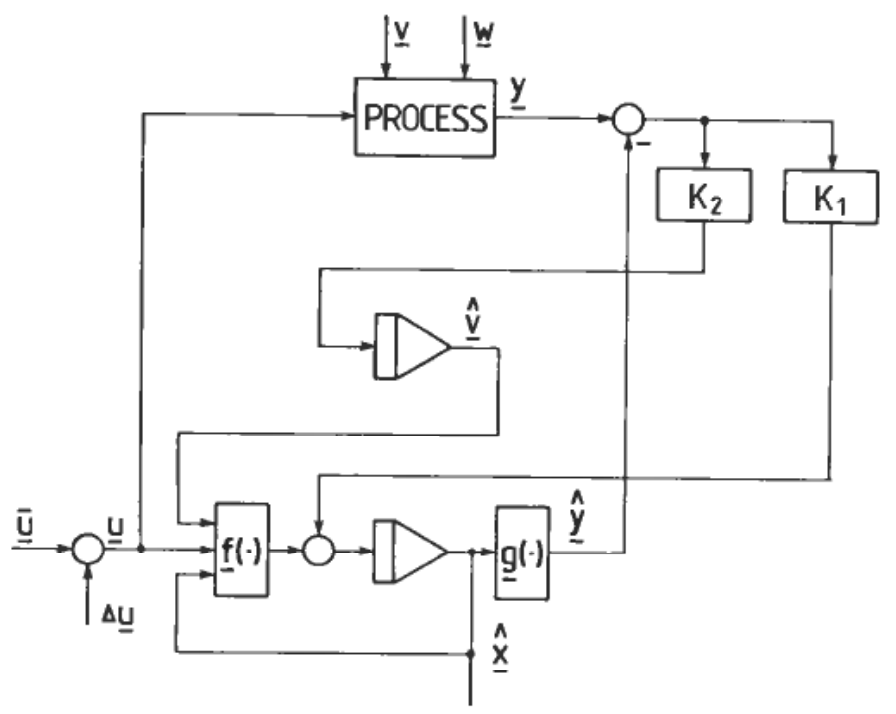

Figure 2. Block-diagram of a state estimator.

and it is assumed that $\Delta v$ is white noise with zero mean and known covariance matrix. Furthermore it is assumed that the measurement noise is zero mean white noise with known covariance matrix and that its influence in (3) can be regarded as additive.

Calculation of the estimator gain matrices $\left(K_{1}\right.$ and $\left.K_{2}\right)$ in Fig. 2 is based upon the well established theory of Kalman filtering (Jazwinski 1970). For certain types of functions $(f(\cdot)$ and $g(\cdot))$ it may be necessary to consider second order compensation of the estimator to avoid biased estimates (see Henriksen 1980), but this detail is omitted here.

According to the above problem formulation the desired control vector is one that maximizes (or minimizes) an objective functional which is the mean of $L_{2}(x, u)$. A common choice of $L_{2}(\cdot)$ is the quadratic form

$$
L_{2}(x, u)=\Delta x^{\mathrm{T}} Q \Delta x+\Delta u^{\mathrm{T}} P \Delta u
$$

where $\Delta x$ and $\Delta u$ represent deviations caused by $\Delta v$. Thus the control vector will consist of two components

$$
\boldsymbol{u}=\overline{\boldsymbol{u}}+\Delta \boldsymbol{u}
$$

where $\bar{u}$ is derived from the steady state optimization of $L_{1}(\bar{x}, \bar{u})$ and $\Delta u$ is derived from (5).

The algorithms for generating $\Delta u$ are well known in the theory of LinearQuadratic-Gaussian optimal control (LQG-control) (Athans 1971).

\section{The quasi-dynamic optimal control strategy}

Figure 3 is a block diagram of the quasi-dynamic optimal control strategy (QDOC) which is a combination of the LQG-strategy generating $\boldsymbol{u}$ and a steadystate profit optimization strategy (SSO) generating $\bar{u}$.

In the LQG-strategy based upon (5), the perturbation $\Delta x$ of the state vector around a slowly varying mean is needed. Figure 3 shows a multivariable highpass 


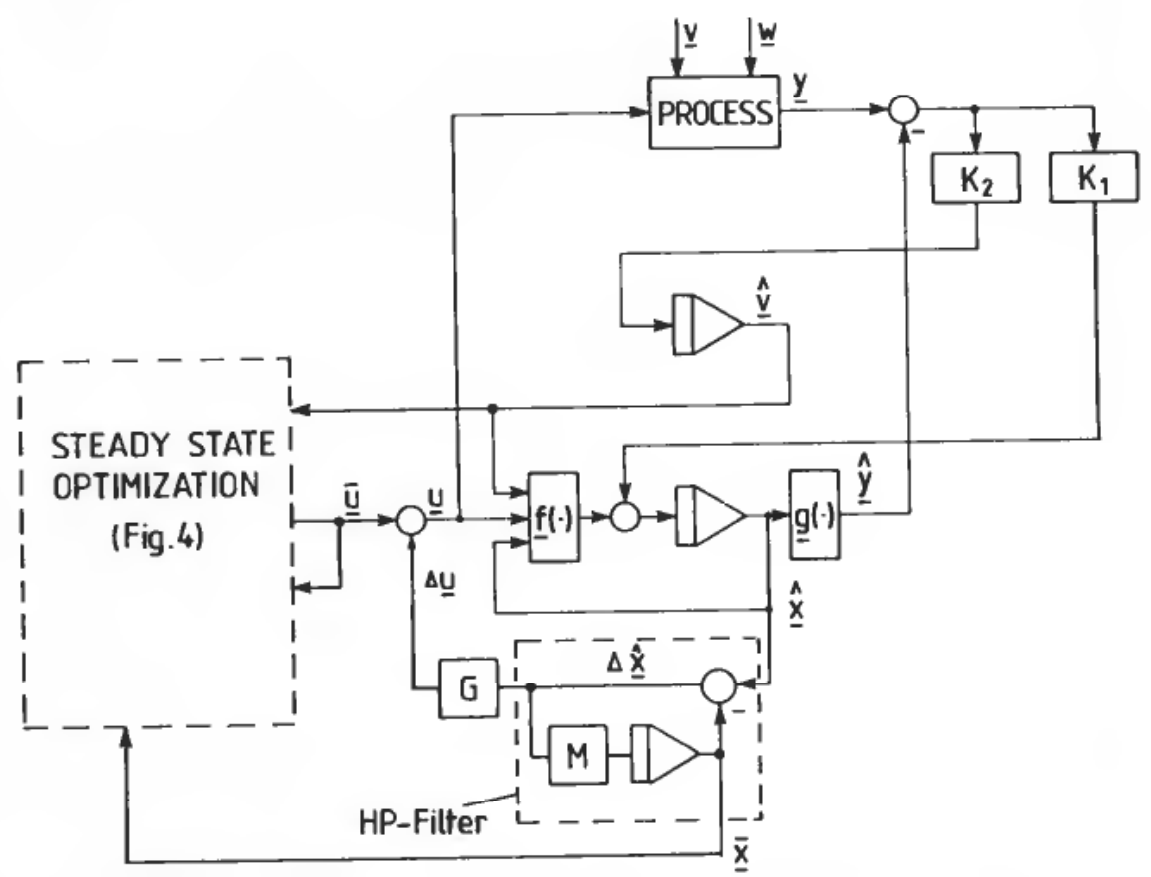

Figure 3. The quasi-dynamic optimal control strategy (QDOC).

filter which extracts $\Delta x$ from $x$ leaving the mean value $\bar{x}$. The feedback control matrix $G$ should in general be made state and control dependent $(G(\bar{x}, \bar{u}))$, but in some cases may be approximated by a constant matrix.

The matrix $M$, appearing in the multivariable highpass filter is diagonal with elements corresponding to the bandwidths as desired. For simplicity it may well be assumed that these bandwidths are identical so that the elements of $M$ become identical. Furthermore the bandwidths will be strongly related to the speed of convergence of the steady state optimization system shown in Fig. 4 and to be described in the next section.

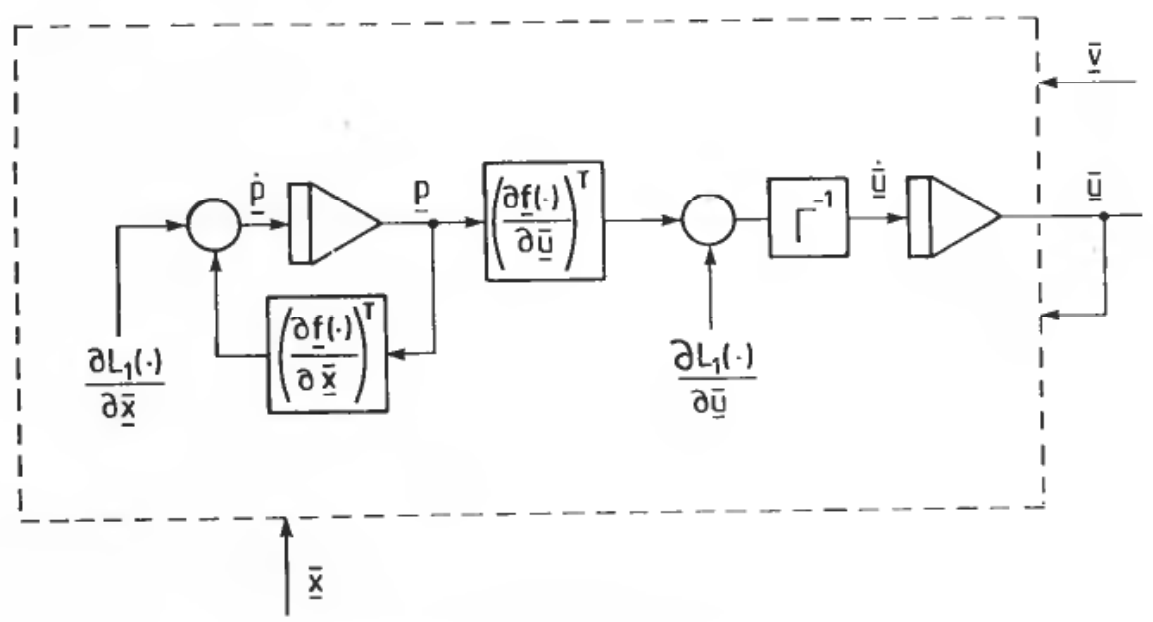

Figure 4. The steady state optimizer (SSO). 


\section{The steady state optimization algorithm}

According to the problem formulation, the slowly varying component (iu) of the control vector must be chosen in such a way as to maximize (or minimize) the objective function $L_{1}(x, u)$. Since the mean values of $\Delta x$ and $\Delta u$ will be zero, the optimization should be based upon $L_{1}(\bar{x}, \bar{u})$.

According to the Maximum Principle, dynamic optimal control is determined by maximizing the Hamiltonian

$$
H(x, u)=L_{1}(x, u)+f^{\mathrm{T}}(x, u, v) p
$$

in which $p$ is the co-state determined by the differential equation

$$
\dot{\boldsymbol{p}}=-\frac{\partial H(\cdot)}{\partial \boldsymbol{x}}
$$

In most cases the final value $p\left(t_{2}\right)$ is given as a boundary condition. This equation, together with (2) with given initial conditions, leads to the Two Point Boundary Value Problem.

The optimal control vector $\left(u_{\text {opt }}\right)$ is that which gives $H(\cdot)$ a maximal value. In the unconstrained case $\boldsymbol{u}_{\mathrm{opt}}$ can be determined by the equation derived from

$$
\frac{\partial H(\cdot)}{\partial u}=0
$$

Since we are looking for the steady state solution of (7) as an approximation to the slowly varying case, we must find the solution of

$$
\dot{p}=-\frac{\partial H(\cdot)}{\partial x}=0
$$

Since the solution of (7) in general requires the integration of the equation backwards from a given final value, we now realize that the problem has become very much simpler since we may replace (9) by

$$
\dot{\boldsymbol{p}}=+\frac{\partial H(\cdot)}{\partial \boldsymbol{x}}=0
$$

which yields exactly the same steady state solution as (9).

The difference is that (10) is integrated forwards to find the steady state solution. Actually the $p$ appearing in (10) is only equal to that appearing in (6) and (9) in the steady state, but for convenience the same notation is used.

Applying (6) in (10) yields the differential equation which determines $p$ in the steady state

$$
\dot{p}=\frac{\partial L_{1}(\cdot)}{\partial x}+\left(\frac{\partial f(\cdot)}{\partial x}\right)^{T} p \rightarrow 0
$$

The optimal control is determined by (8) which is solved by 'iteration' by replacing it with the differential equation

$$
\frac{\partial H(\cdot)}{\partial \boldsymbol{u}}=\Gamma \dot{\boldsymbol{u}} \rightarrow \boldsymbol{0}
$$

In (12) $\Gamma$ is a matrix (preferably constant, but often chosen as the Hessian of the system) which assures good convergence of $u$. 
Applying (6) to (12) leads to

$$
\frac{\partial \mathbf{H}(\cdot)}{\partial u}=\frac{\partial L_{1}(\cdot)}{\partial u}+\left(\frac{\partial f(\cdot)}{\partial u}\right)^{\mathrm{T}} p=\Gamma \dot{u} \rightarrow 0
$$

The contents of (11) and (13) constitute the solution to the steady state optimization problem when $\boldsymbol{x}$ is replaced by $\overline{\boldsymbol{x}}$ and $\boldsymbol{u}$ by $\overline{\boldsymbol{u}}$. The solution is shown in the block diagram of Fig. 4. The following functions appear in this diagram:

$$
\begin{aligned}
& \frac{\partial L_{1}(\cdot)}{\partial \bar{x}}: \begin{array}{l}
\text { a known function of } \bar{x}, \bar{u} \text { and the parameters } \\
\text { of the objective function (prices etc.) }
\end{array} \\
& \frac{\partial L_{1}(\cdot)}{\partial \bar{u}}: \begin{array}{l}
\text { a known function of } \bar{x}, \bar{u} \text { and the parameters } \\
\text { of the objective function }
\end{array}
\end{aligned}
$$

$$
\begin{aligned}
& \frac{\partial f(\cdot)}{\partial \bar{x}} \text { : a known function of } \bar{x}, \bar{u} \text { and } \bar{v} \\
& \frac{\partial f(\cdot)}{\partial \bar{u}} \text { : a known function of } \bar{x}, \bar{u} \text { and } \bar{v} \text {. }
\end{aligned}
$$

If the matrix $\partial f(\cdot) / \partial \boldsymbol{x}$ is non-singular, (11) may be solved directly yielding the steady state value of $p$

$$
p=-\left(\frac{\partial f(\cdot)}{\partial x}\right)^{-\mathrm{T}} \frac{\partial L_{1}(\cdot)}{\partial x}
$$

Applying the solution of (14) to (13) leads to the block diagram of Fig. 5.

Developing a closed expression for

$$
\left(\frac{\partial f(\cdot)}{\partial x}\right)^{-T}
$$

may however be difficult in some cases. Therefore the original form of (11) is recommended even though the solution of (14) may exist in principle.

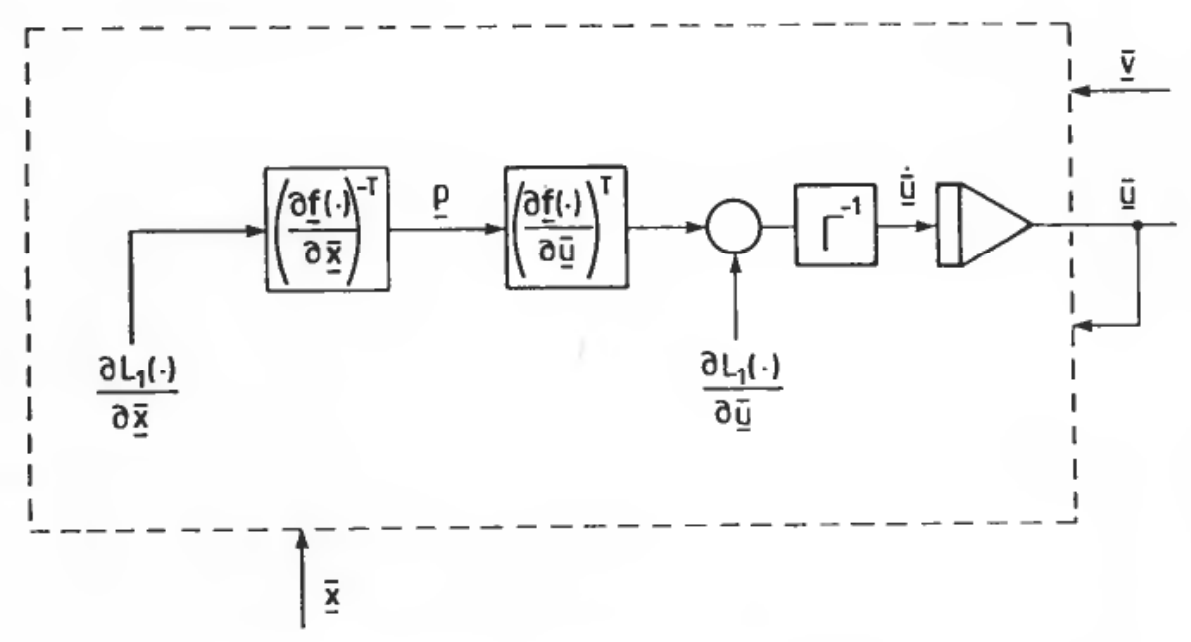

Figure 5. The steady state optimizer when $\left(\frac{\partial f(\cdot)}{\partial \bar{x}}\right)^{\mathrm{T}}$ is singular. 
A common case which makes the matrix $\partial f(\cdot) / \partial x$ singular, is that the process model has open integrators; i.e., no inherent self-regulation. In such a case the solution shown in Fig. 4 must be used. Open integrators will then also appear in the block for determining $\boldsymbol{p}$. This may be interpreted as the application of controllers with integral action (i.e. I-controllers or PI-controllers).

The above derivation of the strategy for determining the optimal steady state control vector $(\bar{u})$ does not take into consideration questions of stability of the resulting feedback loops. These questions must be dealt with separately using standard feedback control theory. For instance in order to arrive at a stable system, the multiple open integrators employed in (12) could be replaced by multiple PIcontrollers which would yield the same steady state solution. Further discussion of methods for ensuring stability in the optimization loops appears in the next section.

\section{Examples}

Some simple examples will illustrate the principles presented in the preceding paragraphs.

\section{Example 1}

Consider the process shown in Fig. 6. It consists of a stirred tank into which there are two controlled flows $\left(u_{1}\right.$ and and $\left.u_{2}\right)$ and from which one flow $\left(v_{3}\right)$ is drawn through a pump. The two incoming flows are chemical components which react in a simple manner. Component 1 is assumed to be acid with a concentration $c_{1}=v_{1}$ and component 2 is a base with the concentration $-c_{2}=v_{2}$. When these components are mixed, they will produce a liquid solution whose concentration $\left(x_{2}\right)$ we will regard as a state variable to be controlled. Since the concentrations of the two components are uncontrollable and may vary, they are regarded as disturbances.

The mass balance in the tank determines the level $\left(x_{1}\right)$ of liquid, which is to be controlled. The system is described by two differential equations

$$
\begin{aligned}
& \dot{x}_{1}=\frac{1}{A}\left(u_{1}+u_{2}-v_{3}\right) \\
& \dot{x}_{2}=\frac{1}{V}\left(v_{1} u_{1}-v_{2} u_{2}-v_{3} x_{2}\right)
\end{aligned}
$$

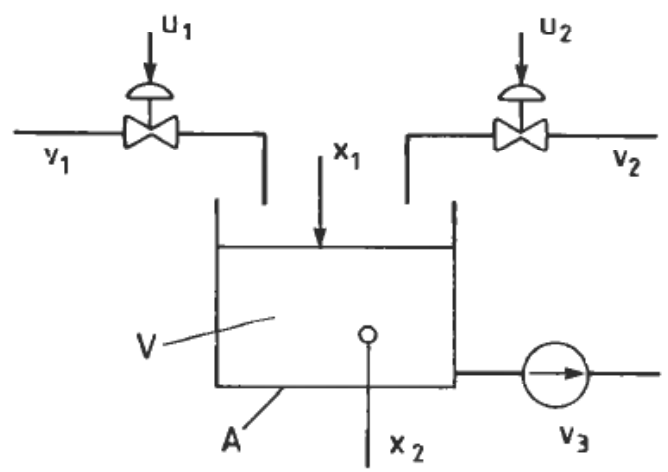

Figure 6. A simple mixing process. 
in which

$A$ : cross-section of tank

$V$ : nominal volume of liquid in tank (assumed constant)

In this case we assume that both state variables $\left(x_{1}, x_{2}\right)$ are directly measurable and that we are not particularily interested in the high frequency control referred to previously as LQG-control. We will only consider the steady state optimization strategy.

Many different types of objective functions $\left(L_{1}(\cdot)\right)$ can be formulated for this simple process. One such function may have the form

$$
L_{1}(\cdot)=\alpha_{1}\left(x_{1}-x_{10}\right)^{2}+\alpha_{2}\left(x_{2}-x_{20}\right)^{2}+\beta_{1} v_{1} u_{1}-\beta_{2} v_{2} u_{2}
$$

$\alpha_{1}$ expresses the penalty associated with the derivation of the level in the tank from a reference level $\left(x_{10}\right) \cdot \alpha_{2}$ similarly expresses the penalty associated with the deviation of the concentration of the mixture from a prescribed concentration $\left(x_{20}\right)$. The cost of component 1 is assumed proportional to a factor $\beta$, its concentration $v_{1}$ and its flowrate $u_{1}$. A similar relationship is assumed for component 2 .

From (16)-(17) we derive the following expressions

$$
\begin{gathered}
\left(\frac{\partial f(\cdot)}{\partial x}\right)^{\mathrm{T}}=\left[\begin{array}{rr}
0 & 0 \\
0 & -\frac{v_{3}}{V}
\end{array}\right] \\
\left(\frac{\partial f(\cdot)}{\partial u}\right)^{\mathrm{T}}=\left[\begin{array}{ll}
\frac{1}{A} & \frac{1}{A} \\
\frac{v_{1}}{V} & -\frac{v_{2}}{V}
\end{array}\right]^{\mathrm{T}}=\left[\begin{array}{ll}
\frac{1}{A} & \frac{v_{1}}{V} \\
\frac{1}{A} & -\frac{v_{2}}{V}
\end{array}\right] \\
\frac{\partial L_{1}(\cdot)}{\partial x}=\left[\begin{array}{c}
2 \alpha_{1}\left(x_{1}-x_{10}\right) \\
2 \alpha_{2}\left(x_{2}-x_{20}\right)
\end{array}\right] \\
\frac{\partial L_{1}(\cdot)}{\partial u}=\left[\begin{array}{r}
\beta_{1} v_{1} \\
-\beta_{2} v_{2}
\end{array}\right]
\end{gathered}
$$

These equations, applied to the steady state optimization strategy illustrated in Fig. 4, lead to Fig. 7 which is a control diagram combined with an elementary block diagram of the process. As can easily be seen, the upper loop contains three open integrators whereas the lower loop has one open integrator. Furthermore, in the controller there is cross coupling between the upper and lower loops. If the disturbances $\left(v_{1}, v_{2}, v_{3}\right)$ are measurable, they should be used directly in the proper computing blocks in the controller of Fig. 7. If they are not measurable, they should be estimated in a scheme similar to that of Fig. 2 and the estimates used instead of measurements.

In Fig. 7 dashed lines indicate modifications to the steady state state optimization algorithm which will assure stability of the system. Each open integrator in the controller is bypassed with a proportional branch, the gain of which must be selected so as to assure stability. The number of parameters $(N)$ to be selected, is determined by

$$
N=2 r+2 q
$$




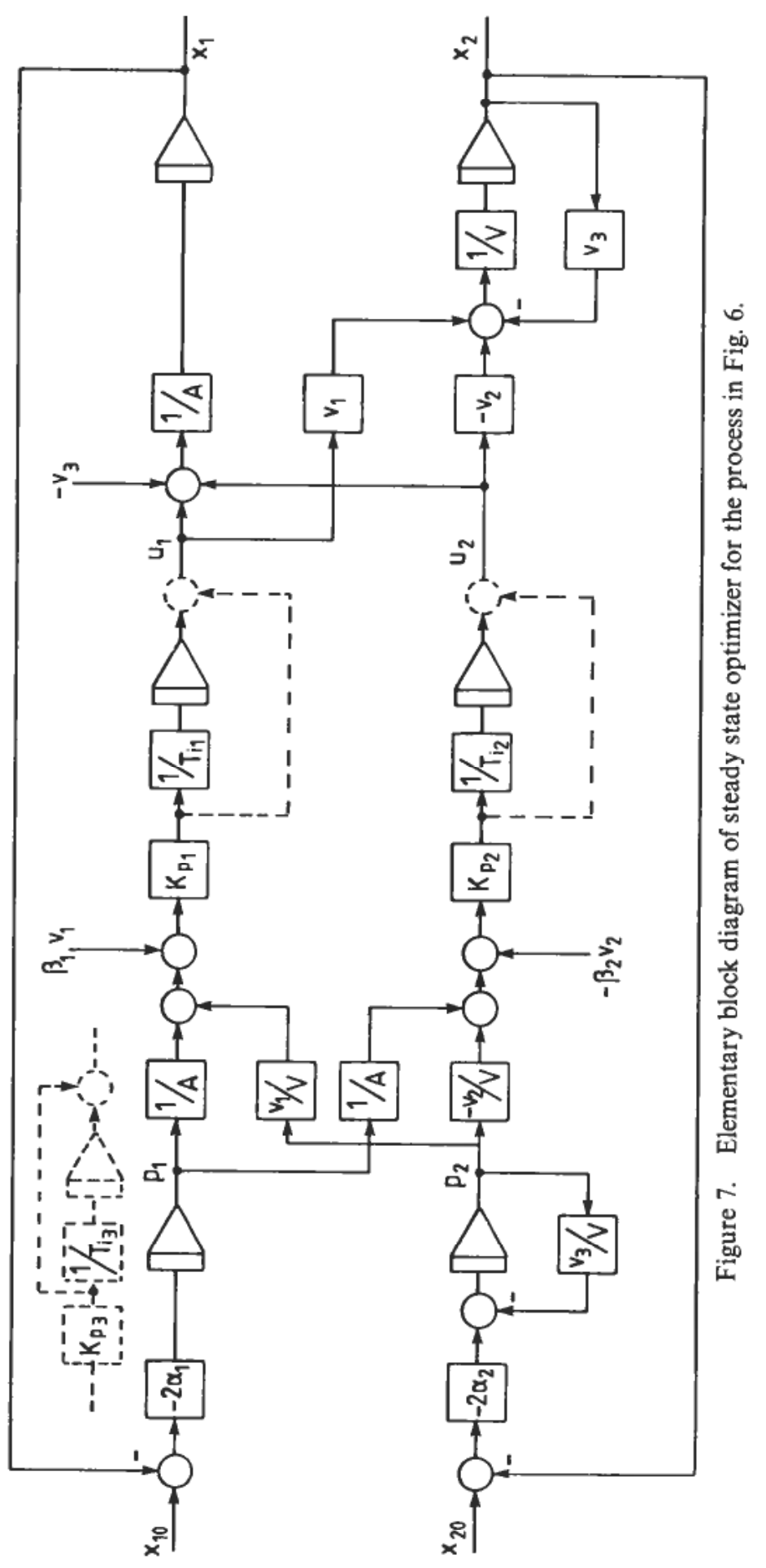


where $r=\operatorname{dim}(u)$ and $q$ is the number of eigenvalues of the matrix $\partial f(\cdot) / \partial x$ with value zero.

The matrix $\Gamma^{-1}$ appearing in the block-diagram of Fig. 4 is seen to have been made diagonal in the block-diagram of Fig. 7. This means that the system of Fig. 7 does not necessarily have the most efficient convergence. A full matrix instead of the diagonal elements $K_{p 1}$ and $K_{p 2}$ will facilitate more rapid convergence, but makes the system more complex.

The transfer-matrix of the open loop multivariable system shown in Fig. 7, will become

$$
H_{0}(s)=-2 H_{u}(s) H_{g}(s) H_{u}^{\mathrm{T}}(s)\left[\begin{array}{cc}
\alpha_{1} & 0 \\
0 & \alpha_{2}
\end{array}\right]
$$

in which

$$
H_{u}(s)=\left(s I-\frac{\partial f(\cdot)}{\partial x}\right)^{-1} \frac{\partial f(\cdot)}{\partial u}
$$

which is the transfer matrix of the process and

$$
H_{g}(s)=\left[\begin{array}{cc}
h_{g 1}(s) & 0 \\
0 & h_{g 2}(s)
\end{array}\right]
$$

where $h_{g 1}(s)$ and $h_{g 2}(s)$ are the controllers solving (12).

Applying the models of the example (23) will become

$H_{0}(s)=-2\left[\begin{array}{c:c}\left.\frac{\alpha_{1}}{A^{2} s^{2}}\left[h_{g 1}(s)+h_{g 2}(s)\right)\right] & \frac{\alpha_{2}}{A V s\left(s+\frac{v_{3}}{V}\right)}\left(v_{1} h_{g 1}(s)-v_{2} h_{g 2}(s)\right) \\ \hdashline \frac{\alpha_{1}}{A V s\left(s+\frac{v_{3}}{V}\right)}\left(v_{1} h_{g 1}(s)-v_{2} h_{g 2}(s)\right) & \frac{\alpha_{2}}{V^{2}\left(s+\frac{v_{3}}{V}\right)^{2}}\left(v_{1}^{2} h_{g 1}(s)+v_{2}^{2} h_{g 2}(s)\right)\end{array}\right]$

From (26) it is seen that by making

$$
h_{g 2}(s)=\frac{v_{1}}{v_{2}} h_{g 1}(s)
$$

$H_{0}(s)$ will become a diagonal matrix.

$$
H_{0}(s)=-2\left[\begin{array}{c:c}
\frac{\alpha_{1}\left(1+\frac{v_{1}}{v_{2}}\right)}{A s^{2}} h_{g 1}(s) & 0 \\
\hdashline 0 & \frac{\alpha_{2} v_{1} v_{2}\left(1+\frac{v_{1}}{v_{2}}\right)}{V^{2}\left(s+\frac{v_{3}}{V}\right)^{2}} h_{g 1}(s)
\end{array}\right]
$$


When the matrix $H_{0}(s)$ is made diagonal, the upper and the lower part of the system in Fig. 7 are decoupled, thereby making the design of the controllers particularly simple. Since the controller $h_{g 1}(s)$ is required to have an integration, it is seen from (28) that the upper loop will contain three integrations.

If the integrator in the upper left half of Fig. 7 is replaced by a PI-controller (as indicated with dashed lines), and furthermore

$$
h_{g 1}(s)=K_{p 1} \frac{1+T_{i 1} s}{T_{i 1} s}
$$

and $T_{i 3}=T_{i 1}$, the loop transfer matrix will become

$$
H_{0}^{\prime}(s)=-2\left[\begin{array}{c:c}
\alpha_{1}\left(1+\frac{v_{1}}{v_{2}}\right) \frac{K_{p 1} K_{p 3}\left(1+T_{i 1} s\right)^{2}}{A T_{i 1}^{2} s^{3}} & 0 \\
\hdashline 0 & \frac{\alpha_{2} v_{1} v_{2}\left(1+\frac{v_{1}}{v_{2}}\right) K_{p 1}\left(1+T_{i 1} s\right)}{v_{3}^{2} T_{i 1} s\left(1+\frac{V}{v_{3}} s\right)^{2}}
\end{array}\right]
$$

Now it can be seen that the lower loop can be made stable by choosing the parameters $K_{p 1}$ and $T_{i 1}$ of the PI-controllers properly. For instance, choosing $T_{i 1}=$ $V / v_{3}$ and

$$
K_{p 1}=\frac{v_{3}^{2}}{\alpha_{2} v_{1} v_{2}\left(1+\frac{v_{1}}{v_{2}}\right)}
$$

will give a reasonable stability margin in the lower loop. With these parameters, the upper loop will be made stable if the controller gain $K_{p 3}$ is made large enough. For instance choosing

$$
K_{p 3}=10 \frac{\alpha_{2} v_{1} v_{2} A}{\alpha_{1} v_{3}^{2} T_{i 1}}
$$

yields an adequate stability margin.

As can be seen from the above elementary analysis, the parameters of the controllers will be dependent upon the actual cost factors of the objective function and of the disturbances $v_{1}, v_{2}$ and $v_{3}$. If these quantities are only slowly varying (which in this example is a realistic assumption), the simplified derivation of the controllers above is applicable.

\section{Example 2}

To further illustrate the structure of the steady-state optimal control strategy the process

$$
\dot{\boldsymbol{x}}=A \boldsymbol{x}+B \boldsymbol{u}+C \boldsymbol{v}
$$

and the objective function

$$
L_{1}(\cdot)=x^{\mathrm{T}} Q x+u^{\mathrm{T}} P u
$$


are chosen. Assuming in this example that $A$ is non-singular, the steady-state solution of (31) will become

$$
\boldsymbol{x}=-A^{-1}(B u+C v)
$$

Applying (33) to (32) yields

$$
L_{1}(\cdot)=u^{\mathrm{T}} B^{\mathrm{T}} A^{-\mathrm{T}} Q A^{-1} B u+2 u^{\mathrm{T}} B^{\mathrm{T}} A^{-\mathrm{T}} Q A^{-1} C v+v^{\mathrm{T}} C^{\mathrm{T}} A^{-\mathrm{T}} Q A^{-1} C v+u^{\mathrm{T}} P u
$$

The steady state optimal control $(u)$ as a function of the disturbance (v) is determined by

$$
\frac{\partial L_{1}(\cdot)}{\partial u}=2\left[\left(B^{\mathrm{T}} A^{-\mathrm{T}} Q A^{-1} B+P\right) u+B^{\mathrm{T}} A^{-\mathrm{T}} Q A^{-1} C \boldsymbol{v}\right]=0
$$

yielding

$$
u_{\text {opt }}=-\left(B^{\mathrm{T}} A^{-\mathrm{T}} Q A^{-1} B+P\right)^{-1} B^{\mathrm{T}} A^{-\mathrm{T}} Q A^{-1} C v
$$

Applying the quasi-dynamic optimal control strategy of (13) and (14), and shown in Fig. 5, will give a steady state control structure as shown in Fig. 8.

If the matrix $P$ is non-singular, the steady state optimal control from Fig. 8 will become

$$
u_{\mathrm{opt}}=P^{-1} B^{\mathrm{T}} A^{-\mathrm{T}} Q x
$$

which when applied to (31) and assuming $\dot{x}=\boldsymbol{0}$ will give

$$
\boldsymbol{x}_{\mathrm{opt}}=-\left(A+B P^{-1} B^{\mathrm{T}} A^{-\mathrm{T}} Q\right)^{-1} C \boldsymbol{v}
$$

and further by the application of (37)

$$
u_{\text {opt }}=-P^{-1} B^{\mathrm{T}} A^{-\mathrm{T}} Q\left(A+B P^{-1} B^{\mathrm{T}} A^{-\mathrm{T}} Q\right)^{-1} C v
$$

(39) and (36) are identical expressions. This can be shown by applying the Matrix inversion lemma to either of the two expressions and thereby yielding the other. The expression of (36) is the most attractive because it does not require $P$ to be nonsingular.

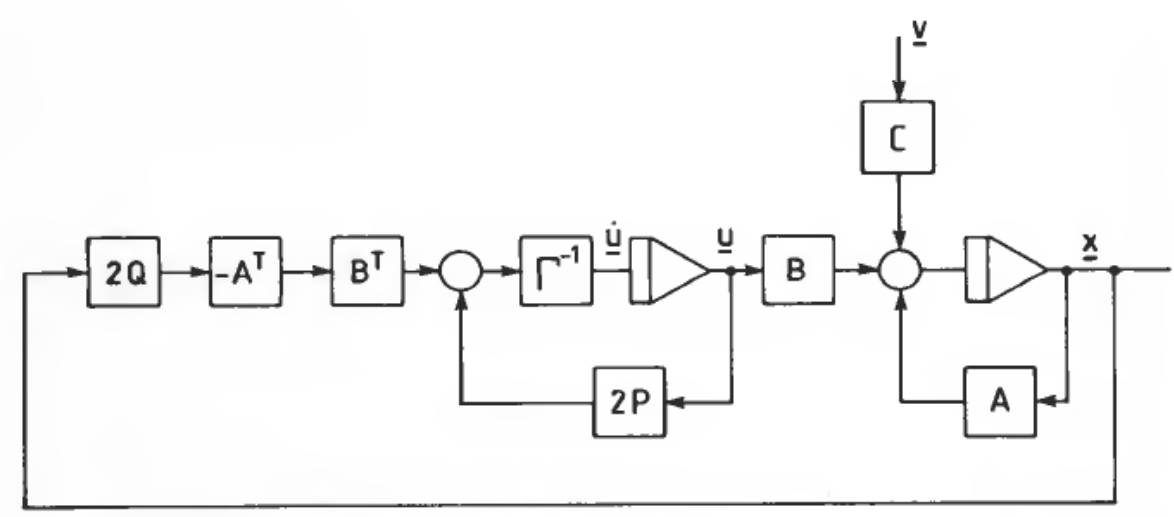

Figure 8. Steady state optimizer for system of (31)-(32). 
The same result can be obtained by observing the two differential equations of Fig. 8 simultaneously

$$
\begin{aligned}
& \dot{x}=A x+B u+C v \\
& \dot{u}=\Gamma^{-1}\left(-2 B^{\mathrm{T}} A^{-\mathrm{T}} Q x+2 P u\right)
\end{aligned}
$$

The steady state solution of (31) which is (33) applied to (40) will in the steady state give (36) directly. This solution applies even if $P=0$ leading to a control structure in the form of multivariable integral feedback control.

The steady state optimal control derived above, is not equal to the ordinary LQG-control because the theory of LQG-control assumes that the disturbances (v) have zero mean. In a real situation this is not at all realistic and may lead to nonoptimal results. Figure 3 indicates how the proper steady state optimization and the LQG-control may be combined.

\section{Penalty functions in optimal control}

Frequently the need arises for expressing amplitude constraints on both control variables and state variables in process control systems. For different reasons related to safety, properties of materials or simply convenience, it may be desired that certain process variables shall stay within given limits.

$$
\begin{gathered}
u_{i, \min } \leq u_{i} \leq u_{i, \max } \\
x_{j, \min } \leq x_{j} \leq x_{j, \max }
\end{gathered}
$$

Actually such sharp constraints are not physically reasonable in many cases and may be regarded as a simplified way of expressing a softer transition of the probability of loss of performance.

An alternative to the type of constraints expressed in (41) and (42) is to include in the objective function terms expressing penalty associated with the violation of given constraints together with the terms expressing profit. One such type of penalty function which has proven to be very effective, is

$$
p(x)=\left(\frac{x-\frac{x_{\max }+x_{\min }}{2}}{\frac{x_{\max }-x_{\min }}{2}}\right)^{N}
$$

$N$ is an even number, the value of which determines the sharpness of the penalty function as illustrated in Fig. 9 for $N=4$ and $N=32$. When penalty functions of the type (43) are added to the objective function $L_{1}(x, u)$, as employed in the derivation of the quasi-dynamic optimal control strategy in the previous sections, the control vector will be forced in such a way as to keep the system within its constraints. Since in the control strategy there will be terms of the form

$$
\frac{\partial p(\cdot)}{\partial x}=\frac{2 N}{x_{\max }-x_{\min }}\left(\frac{x-\frac{x_{\max }+x_{\min }}{2}}{\frac{x_{\max }-x_{\min }}{2}}\right)^{N-1}
$$




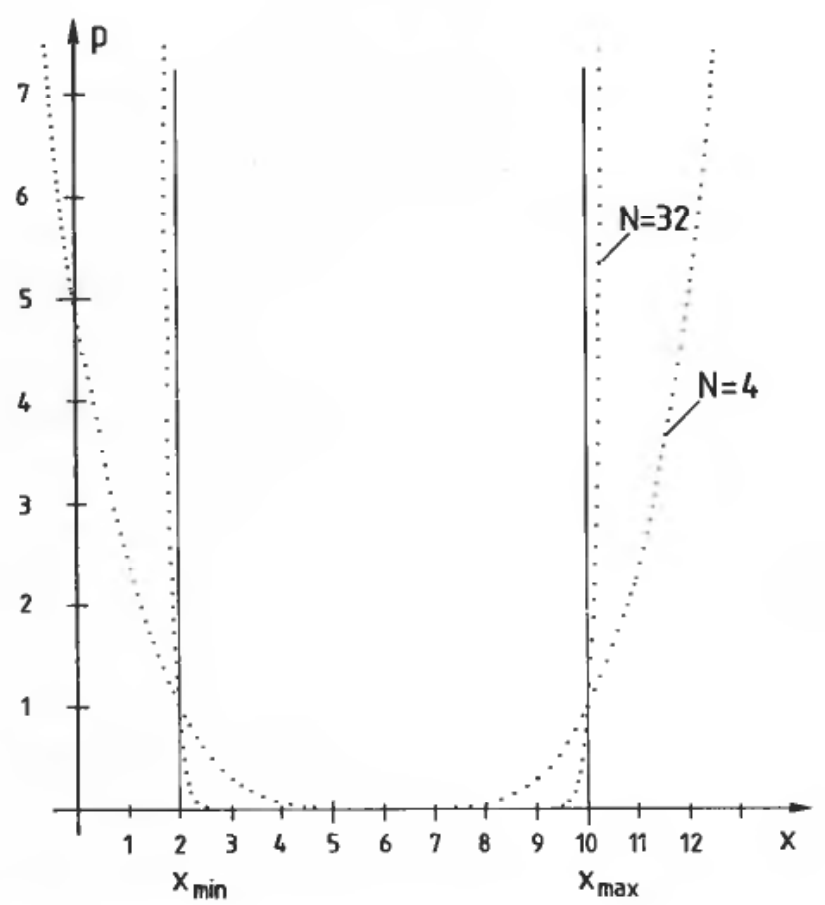

Figure 9. Penalty function as given in (43) for $N=4$ and $N=32$.

and similarly for penalty functions related to $u$, the feedback loops will have very high local gains near the constraints. This may result in problems of stability if no precautions are taken. One solution to this problem is to adjust the gain in the loops at appropriate locations according to expressions of the type

$$
\left(\frac{\partial^{2} p(\cdot)}{\partial x^{2}}\right)^{-1}=\frac{\left(\frac{x_{\max }-x_{\min }}{2}\right)^{2}}{N(N-1)}\left(\frac{\frac{x_{\max }-x_{\min }}{2}}{x-\frac{x_{\max }+x_{\min }}{2}}\right)^{N-2}
$$

Since the expression of (45) will acquire large magnitudes in the domain between the constraints, a certain maximal value should be assigned to the quantity. (45) expresses a simplified version of the statement regarding the matrix $\Gamma$ in (12) which could be chosen as the Hessian of the system.

\section{Conclusions}

In the structural design of process control systems, both the dynamic and the static behaviour of the system play a role. The dynamic structure may be established using available LQG-theory whereas the static structure requires another basis. The paper develops a systematic approach for the structural design of process control systems based upon objective functions which may express the net profit rate of the process. 


\section{REFERENCES}

Athans, M. (1971). The role and use of the stochastic linear-quadratic-gaussian problem in control system design. I.E.E.E. Trans. Automatic Control, 16, 529-552.

Bellman, R., and Kalaba, R. (1965). Dynamic Programming and Modern Control Theory (Academic Press, New York).

HENRIKSEN, R. (1980). A correction of a common error in truncated second-order non-linear filters. Modeling, Identification and Control, 1, 187-193.

JAZWInSKI, A. H. (1970). Stochastic Processes and Filtering Theory. (Academic Press, New York).

Pontryagin, L. S., V. G. Boltyanskil, R. V. Gamkrelidze, and E. F. Mishchenko. (1962). The Mathematical Theory of Optimal Processes. (John Wiley \& Sons, New York). 